LBNL-60369

\title{
Integrated Environmental Assessment Part III: Exposure Assessment
}

\author{
${ }^{1}$ Thomas E. McKone and ${ }^{2}$ Mitchell J. Small \\ ${ }^{1}$ Environmental Energy Technologies Division \\ Indoor Environment Department \\ Lawrence Berkeley National Laboratory \\ Berkeley, CA 94720 \\ ${ }^{2}$ Civil and Environmental Engineering \\ Engineering and Public Policy \\ Carnegie Mellon University \\ Pittsburgh, PA 15213
}

June 2006

This work was supported in part by the US Environmental Protection Agency National Exposure Research Laboratory through Interagency Agreement \#DW-89-93058201-1, through the US Department of Energy under Contract Grant No. DE-AC02-5CH11231. 


\title{
This paper was prepared for submission to the Journal of Industrial Ecology
}

\section{Integrated Environmental Assessment Part III: Exposure Assessment}

\author{
Thomas E. McKone and Mitchell J. Small
}

Human exposure assessment is a key step in estimating the environmental and public health burdens that result chemical emissions in the life cycle of an industrial product or service. This column presents the third in a series of overviews of the state of the art in integrated environmental assessment - earlier columns described emissions estimation (Frey and Small, 2003) and fate and transport modeling (Ramaswami, et al., 2004). When combined, these first two assessment elements provide estimates of ambient concentrations in the environment. Here we discuss how both models and measurements are used to translate ambient concentrations into metrics of human and ecological exposure, the necessary precursors to impact assessment.

Exposure assessment is the process of measuring and/or modeling the magnitude, frequency and duration of contact between a potentially harmful agent and a target population, including the size and characteristics of that population (IPCS, 2001; Zartarian, et al., 2005). Ideally the exposure assessment process should characterize the sources, routes, pathways, and uncertainties in the assessment. Route of exposure refers to the way that an agent enters the receptor during an exposure event. Humans contact pollutants through three routes--inhalation, ingestion, and dermal uptake. Inhalation occurs in both outdoor environments and indoor environments where most people spend the majority of their time. Ingestion includes both water and food, as well as soil and dust uptake due to hand-to-mouth activity. Dermal uptake occurs through contacts with consumer products; indoor and outdoor surfaces; the water supply during washing or bathing; ambient surface waters during swimming or boating; soil during activities such as work, gardening, and play; and, to a lesser extent, from the air that surrounds us. An exposure pathway is the course that a pollutant takes from an ambient environmental medium (air, soil, water, biota, etc), to an exposure medium (indoor air, food, tap water, etc.) and to an exposed individual. Exposure scenarios are used to define plausible pathways for human contact. Recognition of the multiple pathways possible for exposure highlights the importance of a multimedia, multipathway exposure framework.

The magnitude and variation of exposures to environmental contaminants depend largely on two things - (1) the concentrations of contaminants in exposure media and (2) the exposure factors of the target population. So a first step in an exposure assessment is the selection of exposure factors for the targeted population. Human exposure factors include all behavioral, sociological, and physiological characteristics of a population that determine their contact rates with food, air, water, soils, etc. Examples include the inhalation rate (typically $20 \mathrm{~m}^{3} /$ day for an adult), the water ingestion rate (typically 1.4 liters/day), and the soil ingestion rate for children (typically less than $50 \mathrm{mg} / \mathrm{day}$ for 
children 1-6 years old). While these values are representative or conservative, considerable variation in these exposure factors is observed, and distributions characterizing this variability are used in more advanced studies.

In some exposure assessments, a typical or highly exposed individual is used to make scoping or bounding exposure estimates. For example in a regulatory assessment designed to be health protective a "maximally exposed individual" is sometimes the subject of the exposure assessment. This individual might be assumed to spend all of their time at a location of maximum concentration - chained to a fence, eating produce grown at that site, etc. In recent years, however, regulatory assessments have tried to more accurately model the behavior of real people in the environment. There has also been more focus on the variability of exposure. This effort has been aided by a number of comprehensive studies that have used individual personal monitoring in conjunction with ambient measurements. In the United States these included the Total Exposure Assessment Methodology (TEAM) studies and the National Human Exposure Assessment Survey (NHEXAS), while in Europe the Air Pollution Exposure Distributions of Adult Urban Populations in Europe (EXPOLIS) study was conducted (Kousa et al., 2002). These studies have drawn attention to relationships between ambient, indoor, and personal air concentrations for a variety of pollutants, including nitrogen oxides, volatile organic compounds (VOCs), ozone, and fine particulate matter.

Exposure models tend to be chemical-transfer based (tracking chemicals from sources to receptors) or activity based (tracking individuals and concentrations). Specific methods for exposure modeling are reviewed in a number of recent texts (Asante-Duah, 2002; Nieuwenhuijsen, 2003; Ramaswami et al., 2005). Chemical-transfer-based models focus on partitioning and mass transfer (plant uptake, fish bioconcentration, transfer from water to air (showers to indoor air), etc. and then apply contact or intake factors (IPCS, 2000, McKone and MacLeod, 2004). Activity based models focus on time allocations to microenvironments, in which different concentrations, or distributions of concentrations, are modeled to occur. Examples of microenvironments include: outdoors; an individual's home; a home workroom; office or factory; vehicle; and a bar or restaurant. An individual's time allocation in these microenvironments is described by their time-activity pattern. The time-activity pattern keeps track of where people are and, in some cases, what they do while they are there. One-day time-activity patterns for many individuals have been compiled using telephone surveys and these data are available on in databases such as the US EPA's Comprehensive Human Activity Database (CHAD) (http://www.epa.gov/chadnet1/) and the Dutch database of Freijer et al. (1998). Since these databases generally characterize the behavior of individuals on a given day, interesting statistical issues arise when trying to piece together multiple records for longer-term simulations for the population or for a typical individual. Many important exposures occur to an individual when another member of their household undertakes an activity, such as bathing or showering or the use of household chemicals, so methods for tracking co-exposures are also needed for a complete assessment (Wilkes et al., 1996).

A number of models are available for integrating calculations of ambient concentrations and human exposures. One example is the CalTOX model, a multimedia environmental 
model that estimates human exposure through 23 potential exposure pathway scenarios for pollutants in the air, soil, or water (http://eetd.lbl.gov/ied/ERA/; McKone and MacLeod, 2004). These include the ingestion of three classes of food: fruits, vegetables and grains; meat, milk, and eggs; and fish and seafood. The CalTOX model provides a broad assessment of the partitioning of chemicals between the air, water, soil, and biota, and the resulting total exposure. Another type of comprehensive model for exposure is the MENTOR/SHEDS (Modeling Environment for Total Risk studies/Stochastic Human Exposure and Dose Simulation) model. This model has been applied, together with a photochemical model that predicts ambient ozone and fine particle concentrations, to predict total exposures to these pollutants (Georgopoulos et al., 2005).

The focus of Industrial Ecology on issues such as life cycle assessment (LCA) fosters the need for simple but informative metrics of exposure potential. The goal of LCA is to confront and assess impacts over the whole life cycle of a product, from the production of the raw materials to the ultimate disposal of the product. The life-cycle impact assessment (LCIA) process within in LCA is a systematic framework in which emissions are evaluated and interpreted with regard to potential life-cycle health and environmental impacts. The scope of LCIA does not allow for full-scale, site-specific risk assessments. So to provide "effects factors" that can be used as weighting factors for pollutant emissions, LCIA studies rely heavily regional multimedia multipathway exposure models. Generic multimedia exposure models have been used in LCA as "laboratories" for evaluating both the exposure potential of a broad range of pollutants and how exposure potential relates to basic chemical properties (McKone and MacLeod, 2004). Generic versions of both the CalTOX and EUSES models have been used to conduct comparative assessments of fate and exposure in support of LCA, sustainability and comparative risk assessments (Bare et al., 2003; Huijbregts et al., 2005).

To aid in such "generic" assessments in LCA an other studies, the concept of an exposure or intake fraction (iF) has provided a powerful basis for simplification. The intake fraction is that portion of the pollutant mass emitted to the environment that is subsequently taken up by people through inhalation, ingestion, or dermal uptake. Use of this fraction is generally limited to conditions where linear relationships between emissions, ambient concentrations, exposures, and subsequent risk are assumed, however, this is often the case for environmental impact studies conducted for LCIA. Huijbregts et al., 2005) used EUSES-LCA to calculate fate factors and human population iFs for 3933 substances released to freshwater, marine and terrestrial environments. They report iF values averaging $10^{-5}-10^{-8}$ for organics and $10^{-3}-10^{-4}$ for inorganic chemicals, depending on the emission compartment considered. Once the intake fraction is estimated for the emissions from a product in a given use scenario, the resulting transfer factor can be used for similar applications. Levy et al. (2002) report modeled intake fractions between $10^{-8}$ and $10^{-6}$ for particulate air pollution from power plants in the United States. Not surprisingly, the intake fraction for pollutants emitted from vehicles is somewhat higher $\left(\sim 10^{-5}\right)$ and higher still for pollutants emitted indoors, typically $10^{-3}$ to $10^{-1}$ (Lai et al., 2000). Higher intake fractions are also estimated for highly persistent organic compounds that bioaccumulate through the food chain, with intake fractions from dietary intake as high as 0.001 or even 0.01 for compounds such as selected congeners of dioxin 
(Bennett et al., 2002; Pennington et al., 2005). These bounds allow emissions of different types to be broadly categorized in terms of the resulting exposures expected to occur.

When an ambient pollutant is inhaled, ingested, or absorbed, only a portion of the contaminant remains in the body and is delivered to target cells or organs to result in a toxic effect. This process involves the conversion of an exposure to a resulting dose and its associated response. Methods for dose-response analysis are presented in the next column on integrated environmental assessment.

\section{References}

Asante-Cuah, K. 2002 Public Health Risk Assessment for Human Exposure to Chemicals. Kluwer Academic, Dordrecht.

Bare, J.C., G. Norris, D.W. Pennington, and T.E. McKone. 2002."TRACI - The Tool for the Reduction and Assessment of Chemical and Other Environmental Impacts," Journal of Industrial Ecology 6(3-4):49-78.

Bennett, D.H., M.D. Margni, T.E. McKone, and O. Jolliet. 2002. Intake fraction for multimedia pollutants: A tool for life cycle analysis and comparative risk assessment. Risk Analysis 22: 905-918.

Frey, H.C. and M.J. Small. 2003. Integrated environmental assessment, Part 1: Estimating emissions. Journal of Industrial Ecology, 7(1): 9-11.

Freijer, J.I., H.J.T.H. Bloemen, S. de Loos, M. Marra, P.J.A. Rombout, G.M. Steentjies and M.P. van Veen. 1998. Modelling exposure of the Dutch population to air pollution. Journal Hazardous Materials 61: 107-114.

Georgopoulos, P.G., S-W. Wang, V.M. Vyas, Q. Sun, J. Burke, R. Vedantham, T. McCurdy and H. Ozkaynak. 2005. A source-to-dose assessment of population exposures to fine PM and ozone in Philadelphia, PA, during a summer 1999 episode. Journal of Exposure Analysis and Environmental Epidemiology 15: 439-457.

Huijbregts, M.A.J., J. Struijs, M. Goedkoop, R. Heijungs, A.J. Hendriks, D. van de Meent. Human population intake fractions and environmental fate factors of toxic pollutants in life cycle impact assessment. Chemosphere 61(10): 1495-1504

IPCS (2000) Environmental Health Criteria 214 Human Exposure Assessment, Chapter 6 "Human Exposure and Dose Modeling." International Program on Chemical Safety (IPCS), World Health Organization, Geneva.

IPCS (2001) Glossary of Exposure Assessment-Related Terms: A Compilation. Prepared by the Exposure Terminology Subcommittee of the IPCS Exposure Assessment Planning 
Workgroup for the International Programme on Chemical Safety Harmonization of Approaches to the Assessment of Risk from Exposure to Chemicals, November 1, 2001.

Kousa, A., L. Oglesby, K. Doistinen, N. Kunzli and M. Jantunen. Exposure chain of urban air PM2.5- Associations between ambient fixed site, residential outdoor, indoor, workplace and personal exposure in four European cities in the EXPOLIS study.

Atmospheric Environment 26: 3031-3039.

Lai, A.C.K., T. Thatcher and W.W. Nazaroff. 2000. Inhalation transfer factors for air pollution health risk assessment. Journal Air \& Waste Management Association, 50: 1688-1699.

Levy. I.L., S.K. Wolff and J.S. Evans. 2002. A regression-based approach for estimating primary and secondary particulate matter intake fractions. Risk Analysis 22: 895-904.

Marshall, J.D., S-K Teoh and W.W. Nazaroff, "Intake fraction of nonreactive vehicle emissions in US urban areas," Atmospheric Environment, 2005, Vol. 39, pp. 1363-1371.

McKone, T.E., M. MacLeod, (2004) "Tracking Multiple Pathways of Human Exposure to Persistent Multimedia Pollutants: Regional, Continental, and Global Scale Models," Annual Reviews of Environment and Resources, 28:463-492, 2004.

Niewenhuijsen, M.J. (ed) (2003) Exposure Assessment in Occupational and Environmental Epidemiology. Oxford University Press.

Pennington, D.W., M. Margni, C. Amman and O. Jolliet. 2005. Multimedia fate and human intake modeling: Spatial versus nonspatial insights for chemical emissions in Western Europe. Environmental Science \& Technology 39: 1119-1128.

Ramaswami, A., J.B. Milford and M.J.Small. 2004. Integrated environmental assessment, Part II: Modeling fate and transport. Journal of Industrial Ecology, 8(3): 11-13.

Ramaswami, A., J.A. Milford and M.J. Small. 2005. Integrated Environmental Modeling: Pollutant Transport, Fate and Risk in the Environment. John Wiley \& Sons, New York.

Wilkes, C.R., M.J. Small, C.I. Davidson, J.B. Andelman, (1996) Modeling the effects of water usage and co-behavior on inhalation exposures to contaminants volatilized from household water, Journal of Exposure Analysis and Environmental Epidemiology, 6(4): 393-412.

Zartarian, V. T. Bahadori, T. McKone, (2005) "Adoption of an Official ISEA Glossary," Journal of Exposure Analysis and Environmental Epidemiology 15: 1-5. 